\title{
Stellungnahmen der wissenschaftlichen Fachgesellschaften - Zwischenbilanz und weitere Entwicklungen
}

\section{Zusammenfassung}

Rund anderthalb Jahre nach In-Kraft-Treten des Versorgungsstrukturgesetzes zogen die wissenschaftlichen medizinischen Fachgesellschaften (FG) am 25. Juni 2013 anlässlich der zweiten Arbeitstagung zu Stellungnahmen eine Zwischenbilanz. Im Rahmen der Veranstaltung wurden insbesondere Stellungnahmeverfahren zu Gesetzesvorhaben aus dem Bundesgesundheitsministerium (BMG), zu Richtlinien des Robert-Koch-Instituts (RKI), zu Ausarbeitungen des Gemeinsamen Bundesausschusses (G-BA) sowie zu Gesundheitsinformationen des Instituts für Qualität und Wirtschaftlichkeit (IQWiG) thematisiert.

Viele wissenschaftliche Fachgesellschaften haben inzwischen zusätzliche Personal-Strukturen aufgebaut und Prozesse etabliert, um der Herausforderung einer kurzfristigen Erarbeitung von abgestimmten Stellungnahmen gerecht zu werden. Vertreter der Stellungnahme anfragenden Institutionen (G-BA, IQWiG, RKI, AQUA-Institut) antworteten auf Kritik und Anregungen aus Sicht der Fachgesellschaften. Insbesondere werden eine frühzeitigere Einbindung und eine angemessenere Fristsetzung v.a. von BMG und G-BA und eine größere Transparenz im Umgang mit Stellungnahmen gefordert. Die durch die Stellungnahmen erzielten Effekte wurden recht unterschiedlich beurteilt.

\author{
Monika Nothacker ${ }^{1}$ \\ Ina B. Kopp ${ }^{1}$ \\ Wolfgang Müller ${ }^{2}$ \\ Hans-Konrad \\ Selbmann ${ }^{2}$ \\ 1 AWMF-IMWi \\ 2 AWMF, Düsseldorf
}

\section{Text}

\section{Zahlen und Fakten}

Mit Abstand am häufigsten fragte das IQWiG seit Januar 2012 über die Geschäftsstelle der AWMF Stellungnahmen von FG an. Neben Nutzenbewertungen standen hier vor allem Gesundheitsinformationen zur Kommentierung im Vordergrund. Weniger als halb so oft konnte über die AWMF zu Ausarbeitungen des G-BA (Richtlinien, Verordnungen etc.) Stellung genommen werden. Von BMG, AQUA-Institut und RKI lagen jeweils 4-6 Entwürfe zur Kommentierung vor (siehe Abb.1). Die Stellungnahmefristen betrugen zwischen sieben Tagen (Entwurf des Präventionsgesetzes, BMG) und acht Wochen (AQUA-Institut). IQWiG und G-BA gewähren regelhaft eine Frist von 28 Tagen, für die frühe Nutzenbewertung gilt eine Frist von 21 Tagen.

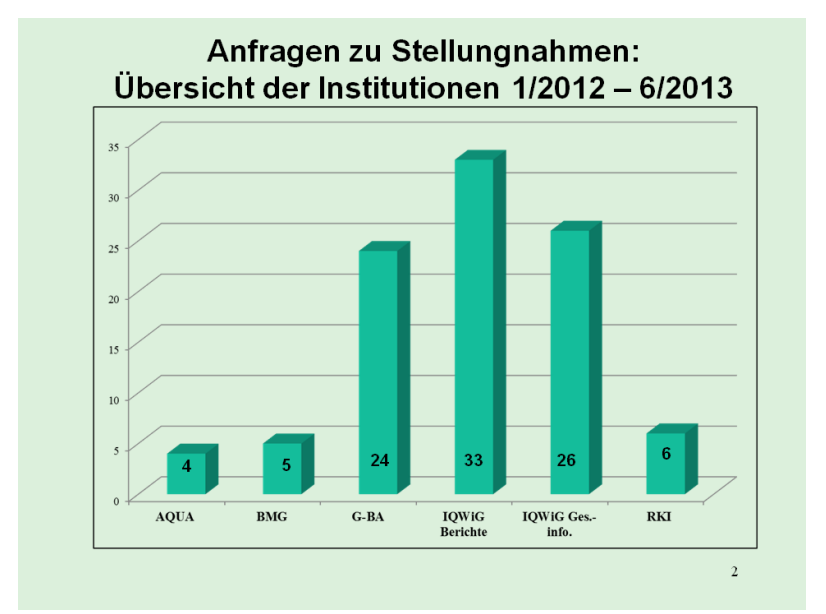

Abbildung 1: Anfragen zu Stellungnahmen - Übersicht der Institutionen 1/2012 - 6/2013

\section{Koordinierung der Stellungnahmen in der AWMF-Geschäftsstelle}

Anfragen zu Stellungnahmen, die bei der AWMF eingehen, werden in der Geschäftsstelle geprüft und nach Beurteilung durch mindestens zwei Personen an die thematisch zuständigen FG versandt. Die AWMF bittet die FG um Rückmeldung, falls keine Stellungnahme erfolgt bzw. um Zusendung der Stellungnahmen. In den Fällen, wo eine eigene Stellungnahme der AWMF erforderlich ist, werden 
die Stellungnahmen der FG berücksichtigt, wenn sie ein bis zwei Tage vor Abgabeschluss vorliegen.

\section{Stellungnahmen zu Gesetzesentwürfen des BMG}

Von der Bundesregierung beim Deutschen Bundestag eingebrachte Gesetzesvorlagen werden in der Regel durch Referenten der Bundesministerien erarbeitet (Referentenentwurf). Der Referentenentwurf wird zur Stellungnahme veröffentlicht. Grundlage dafür ist die gemeinsame Geschäftsordnung der Bundesministerien ( $\S$ 47,3: Rechtzeitige Beteiligung von Ländern, anderen Ressorts und Verbänden. Zeitpunkt, Umfang und Auswahl der Anzufragenden bleiben - soweit keine Sondervorschriften bestehen - dem Ermessen des federführenden Bundesministeriums überlassen [1]). Ob aus den Stellungnahmen Änderungen resultieren, entscheidet zunächst die Bundesregierung, die den Gesetzentwurf als Kabinettsentwurf beschließt. Eine öffentliche Dokumentation dieses Prozesses findet nicht statt. Nach Einbringen des Kabinettsentwurfs in den Bundestag startet das parlamentarische Verfahren mit der ersten Lesung. Abhängig vom festgestellten Klärungsbedarf erfolgt eine Zuweisung an den Gesundheitsausschuss, der entsprechend der Fraktionsstärken paritätisch besetzt ist. Dieser kann eine mündliche Anhörung beschließen[2], anlässlich derer erneut schriftliche Stellungnahmen eingebracht werden können. Die Einladung zur Anhörung erfolgt auf Vorschlag durch die Fraktionen. Nach Beschluss eines Gesetzentwurfs durch den Bundestag besteht die Möglichkeit einer weiteren schriftlichen Stellungnahme an den Bundesrat, in dem das Gesetz anschließend beraten wird. Seit der Föderalismusreform 2006 ist für weniger Gesetzesvorhaben eine Zustimmung desBundesrats erforderlich. Nur wenn diese Zustimmungspflicht besteht, kann der Bundesrat ein Gesetz verhindern [3]. Ist ein Gesetz bereits in Kraft getreten, können Petitionen zu einer Gesetzesänderung an den Bundestag gerichtet werden (siehe auch Abb.2: Stationen des Gesetzentwurfs).

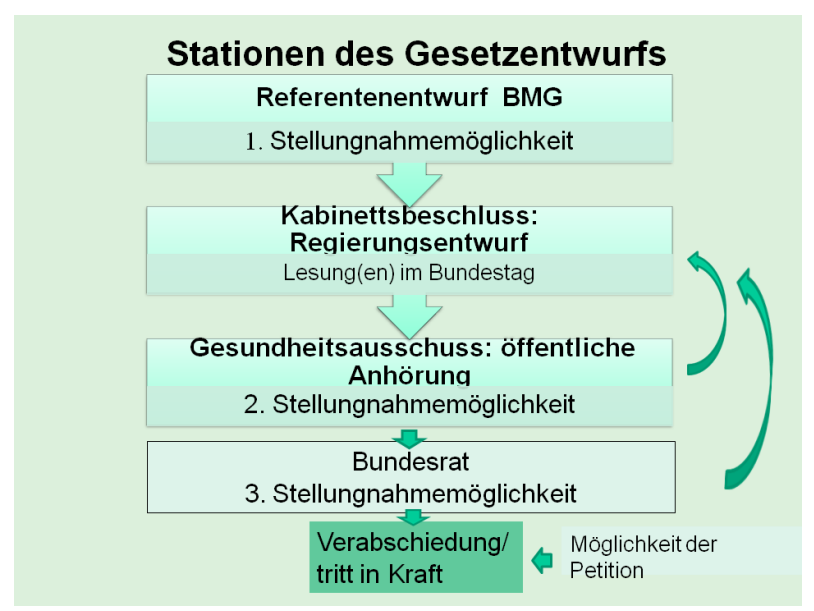

Abbildung 2: Stationen des Gesetzentwurfs

\section{Stellungnahmen zu Richtlinienentwürfen des RKI}

An das RKI konnten bis dato Stellungnahmen zu Richtlinien- und Empfehlungsentwürfen aus der GendiagnostikKommission und der KRINKO (Kommission für Krankenhaushygiene und Infektionsprävention) abgegeben werden. Die ART-Kommission (Kommission Antiinfektiva, Resistenz und Therapie) hat ihre Arbeit erst vor kurzem aufgenommen. Stellungnahmen werden direkt bei den Fachgesellschaften und ergänzend über die AWMF angefragt. Aufgrund der hohen Zahl an zu bearbeitenden Einzelaspekten erfolgte bislang keine öffentliche Würdigung. Insbesondere an die Adresse der KRINKO ging der Wunsch nach einer transparenten und aktuellen Evidenzbasis für die erarbeiteten Empfehlungen.

\section{Stellungnahmen zu Gesundheitsinformationen des IQWiG}

Das IQWiG erstellt Gesundheitsinformationen nach gezielter Recherche auf der Basis systematischer Übersichtsarbeiten oder aber unmittelbar nach Abschluss einer Literaturbewertung im Rahmen von Nutzenbewertungsverfahren. Bei letzteren beziehen sich die Inhalte der Gesundheitsinformation nur auf die Studien und deren Bewertungen durch das IQWiG und nicht auf die abschließende Nutzenbewertung durch den G-BA. Aus diesem Grund veröffentlicht das IQWiG die Gesundheitsinformationen auch bereits vor Abschluss der G-BA Nutzenbewertung. Als sehr positiv wurde die detaillierte und persönliche Würdigung der Stellungnahmen gesehen.

\section{Interne Organisation der Fachgesellschaften zur Erstellung von Stellungnahmen an zwei Beispielen}

Die Deutsche Gesellschaft für Hämatologie und Onkologie (DGHO) engagiert sich mit mehr als 50 Experten vor allem zu Themen der frühen Nutzenbewertung und hat dazu ein Verfahren etabliert, das als vorbildhaft eingeschätzt wurde. Nach dem Screenen der Themen durch Vorstand und Geschäftsstelle werden nach Vorstandsbeschluss kurzfristig jeweils einzelne Verantwortliche für die Bearbeitung benannt. Diese erarbeiten ggf. mit weiteren Experten den ersten Stellungnahmeentwurf. Durch den medizinischen Leiter der Geschäftsstelle erfolgt eine Durchsicht und ggf. eine Modifikation. Anschließend prüft der Vorstand die Stellungnahme, die nach positivem Votum von der Geschäftsstelle veröffentlicht wird (siehe Abb.3 und [4]). Die DGHO hat eine Bewertungstabelle mit ausführlichen Hintergrundinformationen ausgearbeitet, die den Experten die klinische und methodische Bewertung erleichtern soll. 


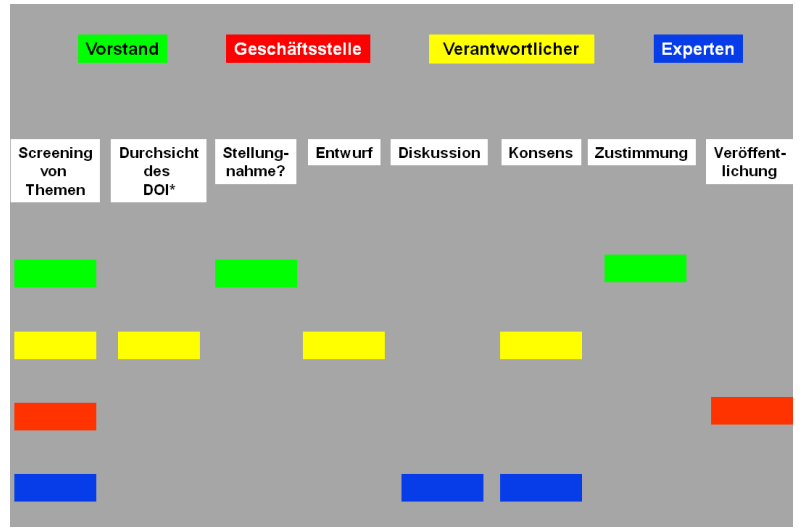

Abbildung 3: Flowchart der DGHO zur Erarbeitung von Stellungnahmen [4]

Die ebenfalls sehr gut strukturierte Vorgehensweise der Deutschen Gesellschaft für Nuklearmedizin (DGN) zeigt, dass auch kleinere FG durch Optimierung des Ressourceneinsatzes die Aufgaben der Stellungnahmen bewältigen können [5]. Die koordinierende DGN-Geschäftsstelle weist nach Beschluss durch den Vorstand Stellungnahmen inhaltlich den mehr als 10 Unterausschüssen zu, die die DGN zu ihren Themen eingerichtet hat. Die Stellungnahmen werden von mehreren Experten erarbeitet und abschließend durch den Vorstand frei gegeben [6].

\section{Stellungnahmen aus der Sicht der FG: Aufwand, Wirkung, Wünsche}

Die Vertreter der FG betonten den personellen und zeitlichen Aufwand, der durch die Anfrage von Stellungnahmen entsteht.

Die kurzen Stellungnahmefristen des IQWIG und des GBA wurden mehrfach als hinderlich thematisiert. Bei Fristen von vier Wochen oder weniger kann eine Abstimmung mit anderen Gesellschaften oder eine frühe Versendung an die AWMF aufgrund der erforderlichen internen Abstimmung kaum realisiert werden. Insbesondere wurde auch die kurze Frist von einer Woche kritisiert, die das BMG für den Gesetzentwurf zum Präventionsgesetz gesetzt hatte. Die AWMF setzt sich für angemessene Fristen bei allen Stellungnahmen anfragenden Institutionen ein.

Diese wurden auch um öffentliche Würdigungen der eingegangenen Stellungnahmen gebeten, wie dies beim IQWiG bereits umgesetzt wird. An die Vertreterin des GBA wurde der Wunsch nach einer aktiven und früheren Ankündigung von Bewertungsverfahren adressiert [7]. Insbesondere zur Festlegung einer adäquaten Vergleichstherapie, aber auch zur Benennung der patientenrelevanten Endpunkte wird eine frühere Beteiligung der Experten aus den wissenschaftlichen FG für notwendig erachtet. Dies könnte im Sinne von Scoping-Workshops realisiert werden, wie sie im Rahmen der Verfahren des AQUA-Instituts erfolgreich etabliert wurden.

Stellungnahmen erfolgen in der Regel zu bereits ausgearbeiteten Vorlagen (Gesetze, Richtlinien, Nutzenbewer- tungen etc.). Dies kann manchmal zu spät sein. Insbesondere bei Gesetzesvorhaben wurde der AWMF empfohlen, früher und direkter mit jenen, die die ersten Entwürfe anfertigen, in Kontakt zu kommen und eine Art „Sensorfunktion" auszuüben, um die den Fachgesellschaften wichtigen Aspekte bereits bei der Entstehung der ersten Entwürfe anzusprechen.

Angesprochen wurden auch aufwändige und zum Teil von Institution zu Institution unterschiedliche Erfordernisse für die Erklärung von Interessenkonflikten. Seitens der FG besteht der Wunsch nach einer Harmonisierung der Verfahren und dem Abbau formaler Hürden [8].

Ein weiterer Aufwand für die FG war in der Vergangenheit die Erfüllung der Forderung (vor allem des G-BA) nach der Einreichung von Volltexten der in den Stellungnahmen zitierten Literaturstellen. Die rechtliche Einschätzung der AWMF ergab, dass die anfragenden Institutionen nicht auf die elektronische Übermittlung von urheberrechtlich geschützten Volltexten bestehen können. Falls Volltexte nicht frei verfügbar sind, sind rechtlich Literaturzitate als Belege angemessen [9].

Für die Fachgesellschaften ist die inhaltliche Berücksichtigung ihrer Stellungnahmen oft nicht transparent, so dass die durch die Stellungnahmen erzielten Effekte nicht sicher beurteilt werden können. Im Ergebnis unterstreichen die FG aber die Forderung nach einer stärkeren Berücksichtigung ihrer Expertise.

Die auf der Veranstaltung anwesenden Vertreter der Institutionen (G-BA, IQWIG, RKI, AQUA-Institut) äußerten sich sehr positiv zur Qualität der eingegangenen Stellungnahmen und unterstrichen deren inhaltliche Bedeutung.

\section{Praktische Tipps und Links:}

- Informationen zu Stellungnahmemöglichkeiten für Fachgesellschaften in der AWMF und links zu den Institutionen

www.awmf.org/service/stellungnahmeverfahren.html

- Verfolgung der Veröffentlichung neuer Beratungsthemen des G-BA (einschließlich frühe Nutzenbewertung) www.g-ba.de/institution/service/ ; Rubrik „E-mail-Infodienst“; Kategorie „Bekanntmachung von Beratungsthemen“ (Abonnement empfohlen)

- Verfolgung neuer Projekte, Ausschreibungen und Produkte des IQWiG

www.iqwig.de/ ; Rubrik „Infodienst“ (Abonnement empfohlen)

- Verfolgung neuer Verfahren des AQUA-Instituts zur Qualitätssicherung www.sqg.de/entwicklung/neue-verfahren/index.html

Die AWMF dankt den Referentinnen und Referenten aus den Institutionen und Fachgesellschaften: 
- PD Dr. H. Tönnies, Robert-Koch-Institut (RKI)

- Dr. K. Koch, Institut für Qualität und Wirtschaftlichkeit im Gesundheitswesen (IQWiG)

- Prof. Dr. B. Wörmann, Deutsche Gesellschaft für Hämatologie und Onkologie

- Hr. G. Jonas, Deutsche Gesellschaft für Nuklearmedizin (DGN)

- Fr. PD Dr. P. Lynen Jansen, Deutsche Gesellschaft für Verdauungs- und Stoffwechselstörungen (DGVS)

- Prof. Dr. E. Solomeyer, Deutsche Gesellschaft für Gynäkologie und Geburtshilfe (DGGG)

- Dr. K.-J-Eßer, Deutsche Gesellschaft für Kinder- und Jugendmedizin (DGKJ)

- Fr. Dr. C. Schulz, Deutsche Gesellschaft für Humangenetik (DGH)

- Prof. J. Windeler, Institut für Qualität und Wirtschaftlichkeit (IQWiG)

- Fr. Dr. E. Pfenning, Gemeinsamer Bundesausschuss (G-BA)

- Prof. J. Szecsenyi, AQUA-Institut

Alle Vorträge sind verfügbar unter: www.awmf.org/service/stellungnahmeverfahren/ a $w \mathrm{~m} f-\mathrm{v}$ e $r$ a n s t a l t u n g e n / 2-arbeitstreffen-am-25-6-2013.html [Zugriff am 20.09.2013].

\section{Literatur}

1. Bundesministerium des Inneren (Hrsg). Gemeinsame Geschäftsordnung der Bundesministerien. Stand September 2011. [Online Zugriff am 20.09.2013] URL: http:// www.bmi.bund.de/SharedDocs/Downloads/DE/ Veroeffentlichungen/ggo.pdf?_blob=publicationFile

2. Deutscher Bundestag (Hrsg). Geschäftsordnung des Deutschen Bundestages und Geschäftsordnung des Vermittlungsausschusses. Berlin, 2013.[Online Zugriff am 20.09.2013] URL: http://www.bundestag.de/bundestag/ aufgaben/rechtsgrundlagen/go_btg/index.html

3. Deutscher Bundesrat. Geschäftsordnung des Bundesrates (GO BR) in der Fassung der Bekanntmachung vom 26. November 1993 (Bundesgesetzblatt Teil I Seite 2007), zuletzt geändert durch Beschluss des Bundesrates vom 8. Juni 2007 (Bundesgesetzblatt Teil I Seite 1057) (Bundesrats-Drucksache 310/07 (Beschluss)) [Online Zugriff am 20.09.213] URL: http:// www.bundesrat.de/cln_350/nn_8690/DE/struktur/recht/go/ go-node.html?_nnn=true

4. siehe Vortrag B. Wörmann "Stellungnahmen von Fachgesellschaften" [Zugriff am 20.09.2013] URL: http:// www.awmf.org/fileadmin/user_upload/Service/ Stellungnahmeverfahren/Info-Veranstaltung_2013-06/ Workshop2_Woermann.pdf
5. G. Jonas, L. Öhme, W. Burchert, Mandat(e) für die Nuklearmedizin- Eine Inventur der Ressourcen (Inventory Resources of Nuclear Medicine). Nuklearmedizin 2012; 51: 4754 doi:10.3413/nukmed-2012020002 [Zugriff am 20.09.2013] URL: http://www.awmf.org/fileadmin/user_upload/Service/ Stellungnahmeverfahren/Info-Veranstaltung_2013-06/ Workshop2_Ressourcen-Inventur_Nuklearmedizin.pdf

6. siehe Vortrag G. Jonas "2. AWMF-Arbeitstreffen, 25. Juni 2013, Stellungnahmen der wissenschaftlichen Fachgesellschaften Interne Organisation in der DGN"[Zugriff am 20.09.2013] URL: http://www.awmf.org/fileadmin/user_upload/Service/ Stellungnahmeverfahren/Info-Veranstaltung_2013-06/ Workshop2_Jonas.pdf

7. Vortrag PD Dr. P. Lynen Jansen "Stellungnahmen aus Sicht der DGVS" [Zugriff am 20.09.2013] URL: http://www.awmf.org/ fileadmin/user_upload/Service/Stellungnahmeverfahren/InfoVeranstaltung_2013-06/Workshop2_Lynen.pdf

8. siehe Vortrag Dr. K. Eßer "Stellungnahmen - Aufwand, Wirkung, Wünsche" [Zugriff am 20.09.2013] URL: http://www.awmf.org/ fileadmin/user_upload/Service/Stellungnahmeverfahren/InfoVeranstaltung_2013-06/Workshop2_Esser.pdf

9. Anmerkung: Dies ergibt sich nach Einschätzung der AWMF unmittelbar aus dem Gesetz: $\S \S 15$ Abs. 1 Nr. 2, 17 Abs. 1 und 2 i.V. m. § 53 Abs. 2 Satz 1 Nr. 1 UrhG und §§ 15 Abs. 1 Nr. 1 , 16 Abs. 1, 53a Abs. 1 S. 2 UrhG. URL: http://www.gesetze-iminternet.de/urhg/BJNR012730965.html.

\section{Korrespondenzadresse:}

Dr. med., MPH Monika Nothacker

AWMF-IMWi c/o Philipps-Universität, Karl-von-Frisch-Str. 1, 35043 Marburg

nothacker@awmf.org

\section{Bitte zitieren als}

Nothacker M, Kopp I, Müller W, Selbmann HK. Stellungnahmen der wissenschaftlichen Fachgesellschaften - Zwischenbilanz und weitere Entwicklungen. GMS Mitt AWMF. 2013;10:Doc12.

DOI: 10.3205/awmf000285, URN: urn:nbn:de:0183-awmf0002853

Artikel online frei zugänglich unter

http://www.egms.de/en/journals/awmf/2013-10/awmf000285.shtml

Eingereicht: 14.10.2013

Veröffentlicht: 15.10.2013

\section{Copyright}

(c)2013 Nothacker et al. Dieser Artikel ist ein Open Access-Artikel und steht unter den Creative Commons Lizenzbedingungen

(http://creativecommons.org/licenses/by-nc-nd/3.0/deed.de). Er darf vervielfältigt, verbreitet und öffentlich zugänglich gemacht werden, vorausgesetzt dass Autor und Quelle genannt werden. 